\title{
Feature selection based on differentially correlated gene pairs reveals the mechanism of IFN- $\beta$ therapy for multiple sclerosis
}

\author{
Tao Jin ${ }^{1}$, Chi Wang ${ }^{2}$, Suyan Tian ${ }^{\text {Corresp. } 3}$ \\ ${ }^{1}$ Department of Neurology and Neuroscience Center, The First Hosptial of Jilin University, Changchun, China \\ 2 Department of Biostatistics and Markey Cancer Center, University of Kentucky, Lexington, United States \\ 3 Division of Clinical Research, The First Hosptial of Jilin University, Changchuan, Jilin, China \\ Corresponding Author: Suyan Tian \\ Email address: windytian@hotmail.com
}

Multiple sclerosis (MS) is one of the most common neurological disabilities of the central nervous system. Immune-modulatory therapy with Interferon- $\beta$ (IFN- $\beta$ ) is a commonly used first-line treatment to prevent MS patients from relapses. Nevertheless, a large proportion of MS patients on IFN- $\beta$ therapy experience their first relapse within two years of treatment initiation. Feature selection, a machine learning strategy, is routinely used in the fields of bioinformatics and computational biology to determine which subset of genes is most relevant to an outcome of interest. The majority of feature selection methods focus on alterations in gene expression levels. In this study, we sought to determine which genes are most relevant to relapse of MS patients on IFN- $\beta$ therapy. Rather than the usual focus on alterations in gene expression levels, we devised a feature selection method based on alterations in gene-to-gene interactions. In this study, we applied the proposed method to a longitudinal microarray dataset and evaluated the IFN- $\beta$ effect on MS patients to identify gene pairs with differentially correlated edges (DCEs) that are consistent over time in the responder group compared to the non-responder group. The resulting gene list had a good predictive ability on an independent validation set and explicit biological implications related to MS. To conclude, it is anticipated that the proposed method will gain widespread interest and application in personalized treatment research to facilitate prediction of which patients may respond to a specific regimen. 


\section{Feature selection based on differentially correlated gene pairs}

2 reveals the mechanism of IFN- $\beta$ therapy for multiple sclerosis

3

4 Tao Jin ${ }^{1}$, Chi Wang 2 , and Suyan Tian ${ }^{3 *}$

$5 \quad{ }^{1}$ Department of Neurology and Neuroscience Center, The First Hospital of Jilin University, 1

6 Xinmin Street, Changchun, Jilin, China, 130021

$7 \quad 2$ Department of Biostatistics and Markey Cancer Center, University of Kentucky, 800 Rose St.

8 Lexington, KY, USA, 40536

$9 \quad{ }^{3}$ Division of Clinical Research, The First Hospital of Jilin University, 1 Xinmin Street,

10 Changchun, Jilin, China, 130021

13 Corresponding author:

14 Suyan Tian

15 Division of Clinical Research

16 The First Hospital of Jilin University

171 Xinmin Street

18 Changchun, Jilin, China, 130021

19 email: windytian@hotmail.com 


\section{Abstract}

23 Multiple sclerosis (MS) is one of the most common neurological disabilities of the central nervous 24 system. Immune-modulatory therapy with Interferon- $\beta$ (IFN- $\beta$ ) is a commonly used first-line 25 treatment to prevent MS patients from relapses. Nevertheless, a large proportion of MS patients 26 on IFN- $\beta$ therapy experience their first relapse within two years of treatment initiation. Feature 27 selection, a machine learning strategy, is routinely used in the fields of bioinformatics and 28 computational biology to determine which subset of genes is most relevant to an outcome of 29 interest. The majority of feature selection methods focus on alterations in gene expression levels. 30 In this study, we sought to determine which genes are most relevant to relapse of MS patients on 31 IFN- $\beta$ therapy. Rather than the usual focus on alterations in gene expression levels, we devised a 32 feature selection method based on alterations in gene-to-gene interactions. In this study, we applied 33 the proposed method to a longitudinal microarray dataset and evaluated the IFN- $\beta$ effect on MS 34 patients to identify gene pairs with differentially correlated edges (DCEs) that are consistent over 35 time in the responder group compared to the non-responder group. The resulting gene list had a good predictive ability on an independent validation set and explicit biological implications related to MS. To conclude, it is anticipated that the proposed method will gain widespread interest and application in personalized treatment research to facilitate prediction of which patients may respond to a specific regimen.

Keywords: Multiple sclerosis; differentially correlated edge; longitudinal; IFN- $\beta$ therapy; treatment response; feature selection. 


\section{Introduction}

46 Multiple sclerosis (MS) is an immune-mediated, inflammatory demyelinating disease of the

47 central nervous system (CNS) that affects about 2.3 million people worldwide [1, 2]. The majority

48 of patients are women between 20 and 40 years old, and the ratio of female to male is about 3:1

$49[1,2]$. Depending on the initial course of disease, MS may be categorized as relapsing-remitting

50 or primary progressive disease [1]. Relapsing-remitting MS (RRMS) is the most prevalent form

51 of MS, accounting for $85 \%$ of MS cases. As indicated by its name, it involves relapses followed

52 by series of remission. In the early stage, patients with this type of MS may experience complete

53 recovery or partial sequel; however, approximately $50 \%$ of patients progress over time from to

54 secondary progressive disease, with or without acute relapses. In contrast, primary progressive MS

55 begins with gradual neurologic deterioration from onset $[1,3]$.

56

57 The etiology of MS has not been fully elucidated, however, it is commonly believed to be triggered 58 as an autoimmune response to an interaction between genetic and environmental factors [4]. In the 59 pathogenesis of MS, T helper (Th)1 cells release pro-inflammatory cytokines such as interleukin602 (IL-2), interferon-gamma (IFN- $\gamma$ ) and tumor necrosis factor- $\alpha$ (TNF- $\alpha$ ) and play a crucial role 61 in inflammation of MS. Th17 cells release IL-17 and have some impacts on MS as well. On the 62 other hand, Th2 cells secrete IL-4, -5, and -10 to counter-regulate Th1 and Th17 responses [5]. 63 Interferon-beta (IFN- $\beta$ ) is the first class of treatment. It was approved for relapsing-remitting MS 64 in 1993, and was subsequently approved in Europe for secondary progressive MS with continued 65 relapse. It is thought that IFN- $\beta$ works to combat MS by stabilizing the blood brain barrier and 66 altering relevant cytokines by redirecting pro-inflammatory Th1 and Th17 immune responses into 67 anti-inflammatory $\mathrm{Th} 2$ responses.

There are an increasing amount of studies suggesting that genomic data such as gene expression profiles and genetic variants data may provide insightful clues on the development and molecular subtypes of MS. For example, Parnell et al. [6] tested the expression values of 110 known MS-related genes, and found that expression of the transcription factors (TF) controlling $\mathrm{T}$ and NK cell differentiation, EOMES, TBX21 and other TFs was significantly lower in MS compared to healthy controls. Besides the long-time well-known strong genetic effect of the HLA-DR2 locus, non-HLA variants (such as IL2R, IL7R and IL2R variants) have been implicated. Among them, IL2R and IL7R are focused the most since they are expressed on regulatory T cells which play crucial roles in regulating the immune response in MS patients[4]. 
79 Feature selection is a machine learning strategy of selecting, from among thousands of genes, a

80

81

82

83

84

85

86

87

88

89

90

91

92

93

94

95

96

97

98

99

100

101

102

103

104

105

106

107

108

109

110

111

112

113

114

115

gene signature (subset) that may be relevant for diagnosis of a disease, segmentation of disease subtypes, patient drug response or survival rate prediction, and is becoming routine practice in the fields of bioinformatics and computational biology $[7,8]$. Until now, the majority of available feature selection methods has focused on investigating alterations of gene expression levels. These are referred to as node-centric methods. An alternative strategy is to focus on alterations of relationships (correlation) among genes, which should also bear meaningful information about which genes are likely to be associated with the outcomes of interest. Here, a "node" corresponds to a gene in a gene-to-gene interaction network/graph and an "edge" corresponds to the correlation between a gene pair. As pointed out by Kostka et al. [9], a differential expression analysis focuses on the first moments (i.e., means) and a differential correlation analysis aims at the second moments instead from the prospect of statistics. So far, development of such edge-centric methods is far from being comparable to development of node-centric counterpart (see [9-11] for examples of edge-centric methods).

3

Compared with classic feature selection methods which mainly deal with cross-sectional data, the feature selection process for longitudinal gene expression data is more complicated. This is unsurprising given the fact that longitudinal gene expression data involve more than a single time point and consider both the expression value trajectory over time and their differences between different phenotypes. So far, feature selection methods specifically to handle longitudinal data are far from sufficient, to the best of our knowledge, the method proposed by Sun et al [12] is one of the few edge-centric methods capable of dealing with longitudinal data. Specifically, in this so called differentially expressed network method, an edge is selected if the directly linked gene pair is strongly correlated (the absolute Spearman correlation coefficient is larger than a threshold) in one group while not in the other group. Combining the differentially expressed genes of the two groups builds a differentially expressed network. However, using a threshold to decide the connection status between gene pairs may result in many falsely positive edges in which the difference of correlation coefficients under two different conditions may be marginal.

Motivated by the differentially expressed network method [12], we proposed a bioinformatics procedure to identify gene pairs with differentially correlated edges over time by replacing the difference in connection statuses with that in actual correlation coefficients. Then we applied the proposed method to a longitudinal microarray dataset which evaluates the effect of IFN- $\beta$ on MS patients. Our objective is to further elucidate the underlying therapeutic mechanisms of IFN- $\beta$ and to predict which subset of MS patients will respond to IFN- $\beta$ therapy based on the identified molecular signature.

Peer] reviewing PDF | (2019:09:41487:2:0:NEW 18 Feb 2020) 


\section{Materials and Methods}

118 Experimental data

119 A microarray experiment dataset was used as a training set in this

120 study (GEO repository accession number GSE24427) [13]. We collected longitudinal gene

121 expression profiles from 25 German relapsing-remitting MS patients treated with recombinant

122 IFN- $\beta-1 \mathrm{~b}(250 \mu \mathrm{g}$ every other day) for two years. The chips were hybridized on the

123 Affymetrix hgu133A and B platforms. Only chips hybridized on the

124 Affymetrix hgu133A platform were considered. This platform included

12522,283 probe sets, corresponding to 12,437 unique genes.

127 Gene expression values were measured at five separate time points:

128 before the first, second, 1-month, 12-month, and 24-month injections.

129 Keeping in mind that several patients may have relapsed shortly after

130 the two-year treatment (in which case their gene expression profiles

131 may have undergone some changes similar to changes of non-

132 responders), we restricted the responder category to patients whose

133 first relapse time was more than 5 years (60 months), resulting in 9

134 responders and 9 non-responders fed into the downstream analysis.

136 To validate the predictive performance of the final model, data from the 137 GSE19285 experiment [14] were downloaded from the GEO repository 138 to serve as a validation set. In that study, 24 relapsing-remitting MS 139 patients were injected with intramuscular IFN- $\beta$-1a once a week and the gene 140 expression data were collected at the first, second and fifth injections. Likewise, the chips of 141 that study were hybridized on both the Affymetrix hgu133A and B platform, 142 but only data on the Affymetrix hgu133A platform were considered in 143 this study. By adding the restriction of more than 5-years duration for 144 the first relapse, 21 patients were included in this testing set; 12 were responders 145 and 9 were non-responders. 
147 Pre-processing procedures

148 Raw data (CEL files) of these two experiments were downloaded from 149 the GEO repository, respectively. The expression values were obtained 150 using the fRMA algorithm [15]. The expression summary values were 151 normalized using quartile normalization and then $\log 2$ transformed.

153 Statistical Methods

154 Identification of consistent DCEs

155 The procedure to identify consistently differentially correlated edges 156 (DCEs) comprised three major steps: 1) interactions in String software 157 [16] were used as a reference network (which slightly downsized the 158 number of genes under consideration from 12,437 to 11,502), then 159 Spearman correlation coefficients (SCCs) were calculated separately 160 for the responder group and the non-responder group at each specific 161 time point; 2) when the absolute difference of SCCs between two 162 groups at a specific time point was larger than a pre-determined 163 threshold (i.e., $\mathbf{0 . 6}$, this value may corresponds to two possible situations: either 164 the correlation directions in two groups are the same with in one group 165 being extremely large whereas in the other group being small; or the correlations 166 in two groups are at least moderate but in different directions. In addition, with 167 this step the number of gene pairs under consideration for $p$-value 168 calculation was downsized to a manageable scale), the corresponding 169 gene pair was deemed to be a DCE at that time point; and, 3) the

170 intersection of DCEs across 5 time points was taken and the resulting 171 subset was considered to represent consistently DCEs over time. A

172 flowchart of the procedure is elucidated using a toy example with 5 genes (Figure 173 1).

174

175 Results 


\section{DCEs}

177 In this study, the connection information in the String software was used as a reference. Only when 178 the confidence score of a gene pair is larger than 0.6, these two genes are considered to be 179 connected by an edge. The cutoff value for the absolute difference of SCCs between two groups 180 is set at 0.6 . Using the proposed procedure, 384 consistently differentially correlated edges (DCEs) 181 over five time points were identified. These edges included 510 unique genes. By saying 182 consistently, it is referred to the scenario that these edges were identified to be differentially 183 correlated across all time points (i.e., the overlapped edges among these time points).

185 Using permutation tests and following the strategy proposed by[17] the significance levels of these 186 gene pairs were determined and presented in Table 1 as well. Twenty-two DCEs are deemed to be 187 statistically significant (with a p-value $<0.01$ ), which involve 41 unique genes (Table 1). Using 188 these 41 genes as predictors, a linear support vector machine model was fit to the training set. The 189 resulting model was applied to the validation set (an independent dataset from the training set) to 190 calculate posterior probability of a MS patient being a responder at each time point. With 4 191 responders and 8 non-responders misclassified, a predictive accuracy of $80.95 \%(51 / 63)$ was

192 achieved by this 41-gene signature. Given that demographic heterogeneities may exist between 193 patients in both experiments, and that the collection time points in the two studies differed hugely, 194 with the early period of treatment being concentrated in the second study, this predictive accuracy 195 is fairly good.

\section{Discussion}

197 The GeneCards database [18] indicates that of the 41-gene signature, 23 genes are directly 198 associated to MS. Among these 23 genes, 7 genes (i.e., CXCL9, IL2RA, CXCR3, AKT1, CSF2, 199 IL2RB and GCA) have a confidence score $>2$ (Figure 2). Likewise, a SVM model was fit with 200 these 7 genes as predictors and then applied to the validation set. This results showed that this $7-$ 201 gene signature can achieve a predictive accuracy of $65.08 \%$ (41/63) with 6 responders and 16

202 non-responders misclassified. Furthermore, these seven genes whose mean expression values for 203 the responders and non-responders across time points are diagramed in Figure 3. According to the 204 EDGE method [19], only AKT1 (adjusted $\mathrm{p}=0.003$ ) and CXCL9 (adjusted $\mathrm{p}=0.012$ ) are 205 significantly differentially expressed over time between these two groups. Focusing specifically 206 on the two extreme points, no genes are differentially expressed at the first time point while only 207 CXCL9 is under-expressed in the responder group at the last time point according to Wilcoxon's 208 tests. 
210 Next, a gene-to-gene interaction network of these 7 genes was constructed using the String

211 software and presented in Figure 4. In this figure, we found that these genes were highly connected 212 with all genes but GCA (which is an isolated gene) circling around one another to frame a loop.

213 Interestingly, it was observed that the edges between IL2RA and IL2RB, and the edge between 214 CXCR3 and CSF2 were negatively correlated to each other in the responder group while positively 215 correlated in the non-responder group. Further study is highly desirable to explore if such 216 correlation patterns have any impact on how a MS patient responds to the IFN- $\beta$ treatment.

217

218 How these 7 genes are related to MS was mined by a PubMed literature searching. Since the 219 autoimmune inflammatory process is believed to be essential for the development of MS, it is 220 natural to observe that chemokines and chemokine receptors are involved in the pathogenesis of the disease [20] For instance, a study [21] compared the chemokine receptor - CXCR3 expression by gamma delta $\mathrm{T}$ cells derived from the blood and CSF of MS patients with health controls and observed its expression increased. Another study [22] also suggested significantly different expression of CXCR3 on peripheral blood lymphocytes in MS compared with controls.

Furthermore, CXCL9 is a ligand of CXCR3. A study [23] described two alternatively spliced

variants of the human CXCR3-A receptor, termed CXCR3-B and CXCR3-alt. While human CXCR3-B binds CXCL9, CXCL10, CXCL11 as well as an additional ligand CXCL4, CXCR3-alt only binds CXCL11. A study by Mellergard et al. [24] suggested there was a marked decline in CSF levels of cytokines and chemokines in MS patients treated with natalizumab, thus including chemokines associated with both Th1 (CXCL9, CXCL10, CXCL11) and Th2 (CCL22). However, the chemokine system is complex and consists of numerous ligands and receptors. It is highly unlikely that targeting a particular simple chemokine or chemokine receptor will give beneficial 234 effects.

A recent review [25] stated that in addition to IL7RA, IL2RA is a well-known immunogenetic marker of MS, explaining why IL2RA was ranked on the top of the directly related to MS list given by the GeneCards database[18]. Although the association between IL2RA and MS has been well established, the functional variation is still unknown. It is speculated that the effect of IL2RA on MS should be better described by several SNPs than by a single one [26], and they showed that the set of SNPs rs2256774 and rs3118470 provided a perfect discrimination between the MS patients and controls. Furthermore, there is a potential link between IL2RA and CXCR5 regarding MS. A study in China [27] showed that the genotypes and allele frequency distributions at the loci of IL2RA rs2104286 and rs12722489 were significantly different between the MS and control 
246 increase the onset risk of MS. IL2RA-rs2104286 showed a positive correlation with CXCR5247 rs3922.

Using a meta-analysis, the association of polymorphisms in IL2RB, IL2RA and IL2 with MS were

250

251

252

253

254

255

256

257

258

259

260

261

262

263

264

265

266

267

268

269

270

271

272

273

274

275

276

277

278

279

280

systemically reviewed, in which significant association for IL2RA and IL2 had been justified [28] and the association for IL2RB was deemed to be insignificant. No significant changes in serum IL-2 and soluble IL-2R levels were found between MS patients and controls, either [29].

The expression of CSF2, also known as GMCSF, by human TH cells has been reported to associated with MS disease severity. GMCSF is strongly induced by interleukin 2 (IL2) [30]: an MS-associated polymorphism in IL2RA specifically increased the frequency of GMCSF producing TH cells. The IL2RA polymorphism regulates IL2 responsiveness of naive TH cells and their propensity to develop into GMCSF producing memory TH cells. AKT is a critical mediator of growth factor-induced neuronal survival in the developing nervous system. Survival factors can suppress apoptosis in a transcription-independent manner by activating the serine/threonine kinase AKT1, which then phosphorylates and inactivates components of the apoptotic machinery. AKT activation relies on the PI3K pathway, and AKT1 is recognized as a critical node in the pathway. Furthermore, a recent study [31] found that compared with wild-type mice, AKT1-/- mice exhibited improved experimental autoimmune encephalomyelitis (EAE), a mouse model of MS. At the cellular level, AKT1 seems to inhibit the proliferation of thymogenic regulatory T cells, thereby promoting Ag-specific Th1/Th17 response. Additionally, Barca's study [32] found that IFN- $\beta$ promotes astrocyte survival by stimulating the PI3K/AKT pathway, and thus concluded that the beneficial effect of IFN- $\beta$ in MS may depend partially on its ability to protect astrocytes from apoptotic cell death at the early stage of MS.

As far as GCA is concerned, Martinez et al. [33] examined four SNPs along the IFIH1-GCAKCNH locus, rs13422767, rs2111485, rs1990760 and rs2068330 and reported an association of this locus with MS for the first time. Nevertheless, such an association had not been validated in another study [34]. The authors explained this discrepancy with three possible reasons: different ethnic background between the study population; different study designs used and the sample size issue.

Furthermore, all these 7 genes except AKT1 and IL2RB are IFN-responsive genes according to the Interferome database (http://www.interferome.org/). In summary, further investigation is warranted to examine how IFN- $\beta$ treatment establishes its effects by targeting these 7 genes.

Peer) reviewing PDF | (2019:09:41487:2:0:NEW 18 Feb 2020) 


\section{Conclusions}

283 Our procedure identified consistent DCEs over time for a responder group in comparison with a 284 non-responder group, and the resulting gene signature has explicit biological relevance to MS. 285 Based on simple calculations of SCCs and their corresponding difference between two different 286 phenotypes, our method is easy to comprehend and can be implemented by an entry-level 287 statistician or a clinician. Therefore, we anticipate its widespread application in relevant research 288 areas, to help researchers identify the underlying therapeutic mechanism of a specific regimen (as 289 shown in the Supplementary Materials, there the proposed procedure was applied to analyze the 290 gene expression data of GSE 41846 [35] in which treated MS patients and non-treated MS patients 291 were compared) and predict which patients are more likely to respond.

292 The statistical procedure used in this study has several limitations. First, it excludes genes that are 293 not in overlapping DCEs, therefore, valuable information may be overlooked, especially for the 294 first time point. In the future, a way to use the first time point as the reference may be considered 295 such that the overlap of other time points with this point will become the focus. Second, the training 296 dataset had only 25 samples in total, and to eliminate possible ambiguous results caused by patients 297 having relapses in the first 2-5 years, 7 MS patients were excluded from the downstream analysis. 298 This results in a smaller sample size. A large gene expression experiment with a better study design 299 is highly desirable.

300

301

302 Availability of data and materials

303 Microarray data (accession numbers: GSE24427 and GSE19285) were downloaded from the Gene

304 Expression omnibus (GEO) repository (https:/www.ncbi.nlm.nih.gov/geo/).

\section{Competing interests}

No competing interests have been declared. 


\section{Funding}

309 This study was supported by the Education Department of Jilin Province (grant No. 310 JJKH20190032KJ), the Natural Science Foundation of China (grants No. 81671177 and 311 31401123), and the Natural Science Foundation of Jilin Province Science and Technology

312 Development Plan Project (No. 20190201043JC).

\section{Author's Contributions}

314 Conceived and designed the study: ST. Analyzed the data: ST TJ. Interpreted data analysis and 315 results: CW ST TJ. Wrote the paper: ST CW TJ. All authors reviewed and approved the final 316 manuscript.

317

\section{Acknowledgements}

319 We thank Donna Gilbreath for scientific editing and assistance in manuscript preparation. 


\section{References}

323

324 1. Browne P, Chandraratna D, Angood C, Tremlett H, Baker C, Taylor BV, Thompson AJ. Atlas

325 of multiple sclerosis 2013: a growing global problem with widespread inequity. Neurology.

$326 \quad 2014 ; 83: 1022-4$.

327 2. Brownlee WJ, Hardy TA, Fazekas F, Miller DH. Diagnosis of multiple sclerosis: progress and 328 challenges. Lancet. 2017;389:1336-46.

329 3. Vargas DL, Tyor WR. Update on disease-modifying therapies for multiple sclerosis. Journal of 330 Investigative Medicine. 2017;65:883-91.

331 4. Nafee T, Watanabe R, Fregni F. Multiple sclerosis. In: Neuromethods. 2018. p. 263-295.

332 5. Garg N, Smith TW. An update on immunopathogenesis, diagnosis, and treatment of multiple 333 sclerosis. Brain Behav. 2015;5(9):e00362.

334 6. Parnell GP, Gatt PN, Krupa M, Nickles D, McKay FC, Schibeci SD, Batten M, Baranzine S, 335 Henderson A, Barnett M, Slee M. The autoimmune disease-associated transcription factors 336 EOMES and TBX21 are dysregulated in multiple sclerosis and define a molecular subtype of 337 disease. Clin Immunol. 2014;151 (1):16-24. doi:10.1016/j.clim.2014.01.003.

338 7. Hira ZM, Gillies DF. A review of feature selection and feature extraction methods applied on 339 microarray data. Adv Bioinformatics. 2015;2015:198363.

340 8. Ang JC, Mirzal A, Haron H, Hamed HN. Supervised, unsupervised, and semi-supervised feature 341 selection: A review on gene selection. IEEE/ACM Trans Comput Biol Bioinforma. 2016;13:971342989.

343 9. Kostka D, Spang R. Finding disease specific alterations in the co-expression of genes. 344 Bioinformatics. 2004;20 SUPPL 1:i194-9.

345 10. Carter SL, Brechbühler CM, Griffin M, Bond AT. Gene co-expression network topology 346 provides a framework for molecular characterization of cellular state. Bioinformatics. $347 \quad 2004 ; 20: 2242-50$.

348 11. Fuller TF, Ghazalpour A, Aten JE, Drake TA, Lusis AJ, Horvath S. Weighted gene 349 coexpression network analysis strategies applied to mouse weight. Mamm Genome. 2007;18:46335072.

351 12. Sun SY, Liu ZP, Zeng T, Wang Y, Chen L. Spatio-temporal analysis of type 2 diabetes mellitus 352 based on differential expression networks. Sci Rep. 2013;3:2268. 
353 13. Hundeshagen A, Hecker M, Paap BK, Angerstein C, Kandulski O, Fatum C, Hartmann C, 354 Koczan D, Thiesen HJ, Zettl UK. Elevated type I interferon-like activity in a subset of multiple 355 sclerosis patients: Molecular basis and clinical relevance. J Neuroinflammation. 2012;9:140.

356 14. Hecker M, Goertsches RH, Fatum C, Koczan D, Thiesen HJ, Guthke R, Zettl UK. Network 357 analysis of transcriptional regulation in response to intramuscular interferon- $\beta$-1a multiple 358 sclerosis treatment. Pharmacogenomics J. 2012;12 (2):134-46.

359 15. McCall MN, Bolstad BM, Irizarry RA. Frozen robust multiarray analysis (fRMA). 360 Biostatistics. 2010;11:242-53.

361 16. Franceschini A, Szklarczyk D, Frankild S, Kuhn M, Simonovic M, Roth A, Lin J, Minguez P, 362 Bork P, Von Mering C, Jensen LJ. STRING v9.1: Protein-protein interaction networks, with 363 increased coverage and integration. Nucleic Acids Res. 2013;41:D808-15.

364 17. Anglani R, Creanza TM, Liuzzi VC, Piepoli A, Panza A, Andriulli A, Ancona N. Loss of 365 connectivity in cancer co-expression networks. PLoS One. 2014;9(1): e87075.

18. Safran M, Dalah I, Alexander J, Rosen N, Iny Stein T, Shmoish M, Nativ N, Bahir I, Doniger T, Krug H, SIrota-Madi A, Olender T, Golan Y, Stelzer G, Harel A, Lancet D. GeneCards Version 3: the human gene integrator. Database (Oxford). 2010;2010:baq020.

19. Storey JD, Xiao W, Leek JT, Tompkins RG, Davis RW. Significance analysis of time course microarray experiments. Proc Natl Acad Sci. 2005;102:12837-42.

20. Szczuciński A, Losy J. Chemokines and chemokine receptors in multiple sclerosis. Potential targets for new therapies. Acta Neurol Scand. 2007;115:137-46.

21. Murzenok PP, Matusevicius D, Freedman MS. $\gamma / \delta$ T cells in multiple sclerosis: Chemokine and chemokine receptor expression. Clin Immunol. 2002;103 3 I:309-16.

375

376

377

378

379

380

381

382

383

384

22. Mahad DJ, Lawry J, Howell SJL, Woodroofe MN. Longitudinal study of chemokine receptor expression on peripheral lymphocytes in multiple sclerosis: CXCR3 upregulation is associated with relapse. Mult Scler. 2003;9:189-98.

23. Korniejewska A, Mcknight AJ, Johnson Z, Watson ML, Ward SG. Expression and agonist responsiveness of CXCR3 variants in human T lymphocytes. Immunology. 2011;132:503-15.

24. Mellerård J, Edström M, Vrethem M, Ernerudh J, Dahle C. Natalizumab treatment in multiple sclerosis: Marked decline of chemokines and cytokines in cerebrospinal fluid. Mult Scler. 2010;16:208-17.

25. Leray E, Moreau T, Fromont A, Edan G. Epidemiology of multiple sclerosis. Rev Neurol (Paris). 2016;172:3-13. doi:10.1016/j.neurol.2015.10.006. 
385 26. Babron MC, Perdry H, Handel AE, Ramagopalan S V., Damotte V, Fontaine B, Muller386 Myhsok B, Ebers GC, Clerget-Darpoux F. Determination of the real effect of genes identified in 387 GWAS: The example of IL2RA in multiple sclerosis. Eur J Hum Genet. 2012;20:321-5.

388 27. Xia ZL, Qin QM, Zhao QY. A genetic link between CXCR5 and IL2RA gene polymorphisms 389 and susceptibility to multiple sclerosis. Neurol Res. 2018;40:1040-7. 390 doi:10.1080/01616412.2018.1517110.

391 28. Cavanillas ML, Alcina A, Ñez C, De Las Heras V, Fernández-Arquero M, Bartolomé M, de la 392 Concha EG, Fernandez O, Arroyo R, Matesanz F, Urcelay E. Polymorphisms in the IL2, IL2RA 393 and IL2RB genes in multiple sclerosis risk. Eur J Hum Genet. 2010;18:794-9.

394 29. Ott M, Demisch L, Engelhardt W, Fischer PA. Interleukin-2, soluble interleukin-2-receptor, 395 neopterin, 1-tryptophan and $\beta 2$-microglobulin levels in CSF and serum of patients with relapsing396 remitting or chronic-progressive multiple sclerosis. J Neurol. 1993;241:108-14.

397 30. Hartmann FJ, Khademi M, Aram J, Ammann S, Kockum I, Constantinescu C, Gran B, Piehl 398 F, Olsson T, Codarri L, Becher B. Multiple sclerosis-associated IL2RA polymorphism controls 399 GM-CSF production in human TH cells. Nat Commun. 2014;5:5056.

400 31. Ouyang S, Zeng Q, Tang N, Guo H, Tang R, Yin W, Wang A, Tang H, Zhou J, Xie H, Langdon 401 WY, Yang H, Zhang J. Akt-1 and Akt-2 Differentially Regulate the Development of Experimental 402 Autoimmune Encephalomyelitis by Controlling Proliferation of Thymus-Derived Regulatory $\mathrm{T}$ 403 Cells. J Immunol. 2019;202(5):1441-52.

404 32. Barca O, Ferré S, Seoane M, Prieto JM, Lema M, Señarís R, Arce VM. Interferon beta 405 promotes survival in primary astrocytes through phosphatidylinositol 3-kinase. J Neuroimmunol. 2003;139:155-9.

407 33. Martínez A, Santiago JL, Cénit MC, de las Heras V, de la Calle H, Fernández-Arquero M, 408 Arroyo R, de la Concha EG, Urcelay E. IFIH1-GCA-KCNH7 locus: Influence on multiple 409 sclerosis risk. Eur J Hum Genet. 2008;16(7):861-4.

410 34. Couturier N, Gourraud PA, Cournu-Rebeix I, Gout C, Bucciarelli F, Edan G, Babron MC, 411 Clerget-Darpoux F, Clanet M, Fontaine B, Brassat D. IFIH1-GCA-KCNH7 locus is not associated 412 with genetic susceptibility to multiple sclerosis in French patients. Eur J Hum Genet. 413 2009;17(6):844-7.

414 35. Nickles D, Chen HP, Li MM, Khankhanian P, Madireddy L, Caillier SJ, Santaniello A, Cree 415 BA, Pelletier D, Hauser SL, Oksenberg JR, Baranzine SE. Blood RNA profiling in a large cohort 416 of multiple sclerosis patients and healthy controls. Hum Mol Genet. 2013;22(20):4194-205. 
Figure 1. Flowchart of the proposed procedure. (A) Identification of differentially correlated edges. (B) Generation of consistently differentially correlated edges. Here, a toy-example with 5 genes was used to graphically elucidate the proposed procedure. First, a reference network was created according to the String software. Only the interactions recorded in this software were considered. Then the Spearman correlation coefficients for these gene pairs were calculated for the responder group and the non-responder group, respectively. If the absolute value of the correlation coefficient difference between these two groups is larger than a predetermined cutoff, say, 0.6 , the corresponding edge is deemed as differentially correlated edge (DCE). Those common DCEs across time points are consistently DCEs. Then the corresponding p-values for these DCEs were calculated using permutation tests. Upon the genes involved in the statistically significant DCEs, the biological relevance was investigated.

Figure 2. Venn-diagram showing 41 genes involved in the statistically significant DCEs. Among them, 23 genes are indicated to be directly related to multiple sclerosis, whose symbols are given in the Venn-diagram. 8 of 23 genes have a confidence score $>2$, which are highlighted in red. DCEs: consistently differentially correlated edges.

Figure 3. Mean expression value trajectories of the identified 7-gene list for the responder and the non-responder. (A) For CXCL9. (B) For IL2RA. (C) For CXCR3. (D) For AKT1. (E) For CSF2. (F) For IL2RB. (G) For GCA. Here, R: the responder group; NR: the non-responder 444 group.

Figure 4. Gene-to-gene interaction network of the identified gene list. (A) The responder group. (B) The non-responder group. Here, + stands for the correlation coefficient between the corresponding gene pair is positive; - stands for the correlation coefficient between the corresponding gene pair is negative. 
452

453

454

455

456

457 
Figure 1

Flowchart of the proposed procedure

(A) Identification of differentially correlated edges. (B) Generation of consistently differentially correlated edges. 
A

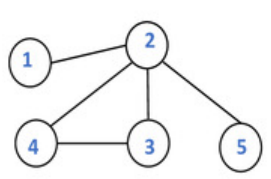

Reference network based on the String software

Correlation matrix

\begin{tabular}{|c|c|c|c|c|c|}
\hline \multicolumn{6}{|c|}{ Responder } \\
\hline gene & 1 & 2 & 3 & 4 & 5 \\
\hline 1 & 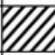 & 0.75 & UIII & MII & $D$ \\
\hline 2 & 0.75 & DII & -0.2 & 0.6 & 0.1 \\
\hline 3 & & -0.2 & & & DII \\
\hline 4 & I & 0.6 & & & ID \\
\hline 5 & 8 & 0.1 & & & A \\
\hline
\end{tabular}

\begin{tabular}{|c|c|c|c|c|c|}
\hline \multicolumn{6}{|c|}{ Non-responder } \\
\hline gene & 1 & 2 & 3 & 4 & 5 \\
\hline \multicolumn{6}{|c|}{${ }^{1}$ Ulla ${ }^{0.6}$ Yllallalla } \\
\hline 2 & 0.6 & II & 0.7 & 0.4 & -0.8 \\
\hline 3 & & 0.7 & & & \\
\hline 4 & C & 0.4 & & & \\
\hline 5 & & & & & \\
\hline
\end{tabular}

> a predetermined cutoff

B
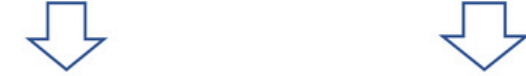

Responder

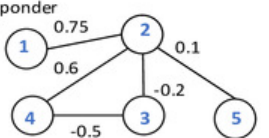

non-responder

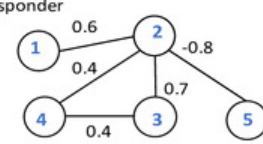

$\sqrt{5}$

Time point: $\mathrm{t}$

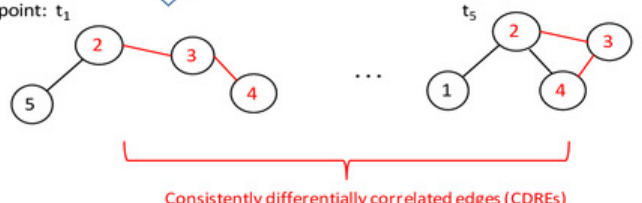

Consistently differentially correlated edges (CDRES)

Unique genes

involved in

these CDREs 
Figure 2

Venn-diagram showing 41 genes involved in the statistically significant DCEs. 
AKT1 RFC4 ADCY3 CROCC DYNC1H1 UBE2D2 CEP290

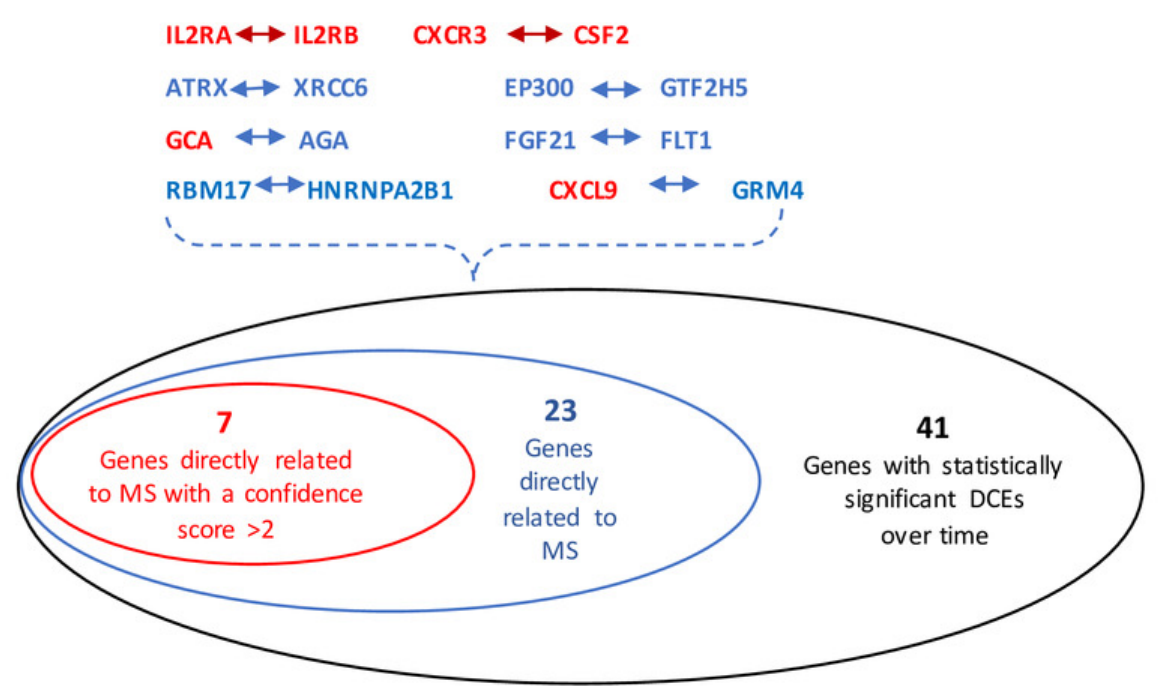


Figure 3

Mean expression value trajectories of the identified 7-gene list for the responder and the non-responder.

(A) For CXCL9. (B) For IL2RA. (C) For CXCR3. (D) For AKT1. (E) For CSF2. (F) For IL2RB. (G) For GCA.
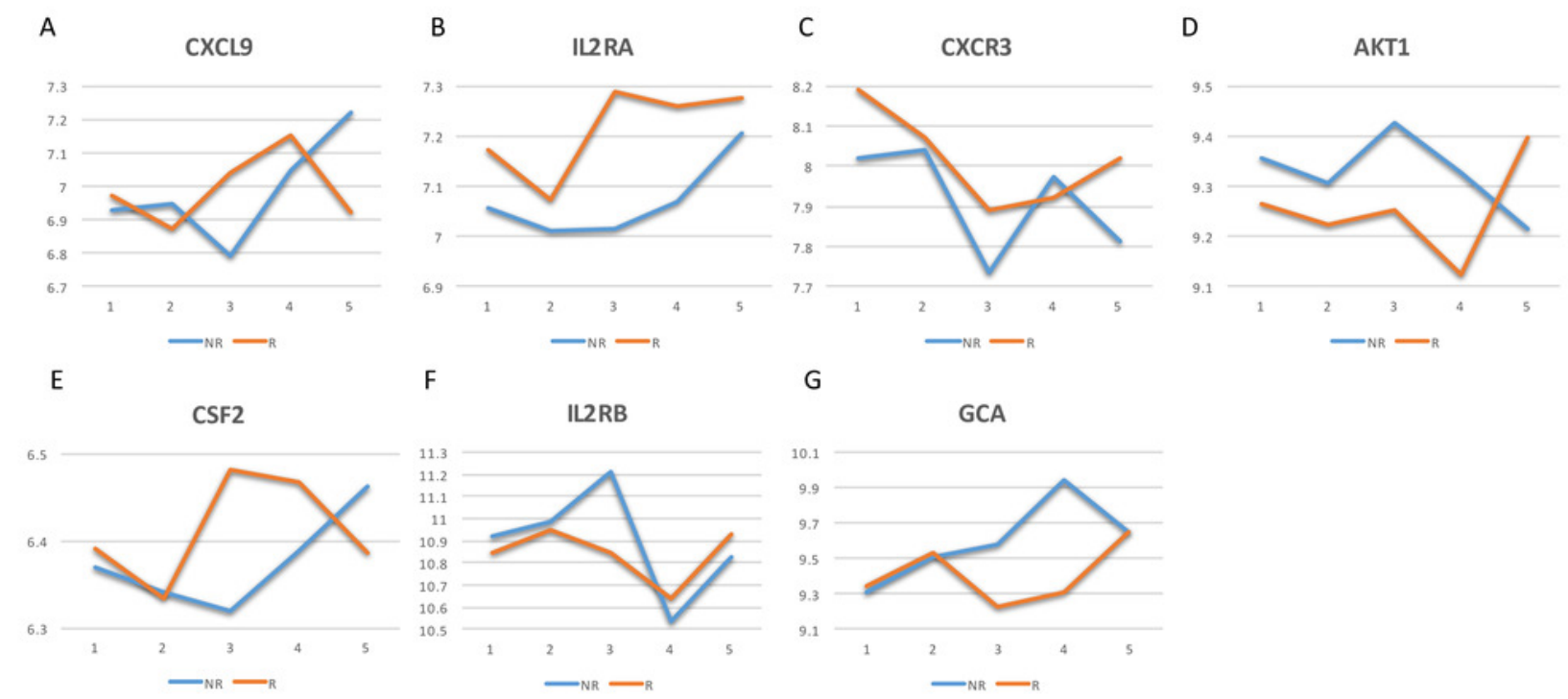
Figure 4

Gene-to-gene interaction network of the identified gene list.

(A) The responder group. (B) The non-responder group.

A. For responders

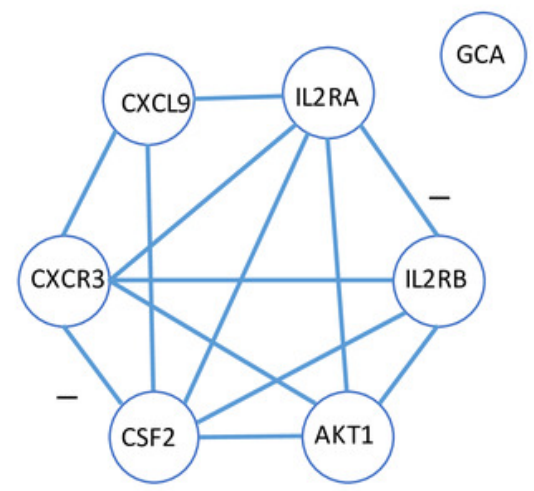

B. For non-responders

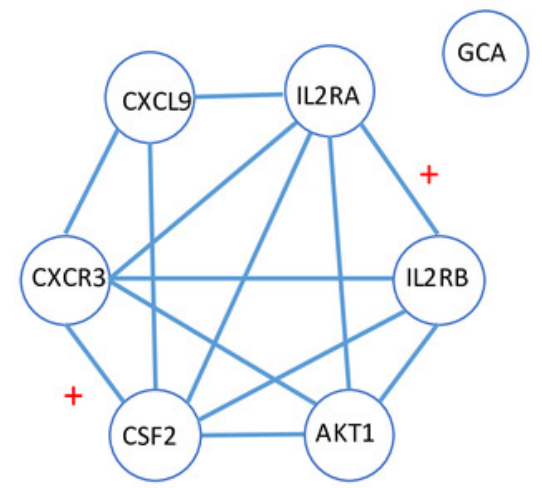




\section{Table $\mathbf{1}$ (on next page)}

Consistent edge differences (network differences) across five time points 


\section{Table 1. Consistent edge differences (network differences) across five time points}

\begin{tabular}{|c|c|c|c|c|c|c|c|c|c|c|c|c|}
\hline \multirow[t]{2}{*}{ Gene1 } & \multirow[t]{2}{*}{ Gene2 } & \multicolumn{2}{|c|}{$\mathrm{T} 1$} & \multicolumn{2}{|c|}{$\mathrm{T} 2$} & \multicolumn{2}{|c|}{ T3 } & \multicolumn{2}{|c|}{$\mathrm{T} 4$} & \multicolumn{2}{|c|}{$\mathrm{T5}$} & \multirow[t]{2}{*}{$\begin{array}{c}\mathrm{p}- \\
\text { value }\end{array}$} \\
\hline & & $\underline{\mathrm{R}}$ & NR & $\underline{\mathrm{R}}$ & NR & $\underline{R}$ & NR & $\underline{R}$ & NR & $\underline{\mathrm{R}}$ & NR & \\
\hline ADCY1 & AKT1 & -0.567 & 0.65 & -0.717 & 0.7 & -0.383 & 0.667 & -0.883 & 0.283 & -0.683 & 0.167 & 0.0001 \\
\hline FKBP10 & HTR5A & 0.633 & $\underline{-0.4}$ & 0.717 & -0.433 & 0.833 & -0.033 & 0.617 & -0.217 & 0.967 & -0.083 & 0.0087 \\
\hline DCAF13 & $\underline{\mathrm{KRR} 1}$ & $\underline{-0.533}$ & $\underline{0.467}$ & $\underline{-0.133}$ & $\underline{0.6}$ & $\underline{-0.6}$ & $\underline{0.35}$ & $\underline{0.533}$ & $\underline{\underline{-0.5}}$ & $\underline{-0.7}$ & $\underline{0.217}$ & $\underline{0}$ \\
\hline ATRX & XRCC6 & 0.233 & -0.533 & 0.283 & -0.55 & -0.75 & -0.117 & 0.517 & -0.467 & 0.567 & -0.5 & 0.0095 \\
\hline CSF2 & CXCR3 & $\underline{-0.1}$ & -0.883 & 0.3 & $\underline{-0.7}$ & -0.033 & -0.783 & $\underline{0.283}$ & -0.6 & $\underline{0.333}$ & $\underline{-0.767}$ & 0.0083 \\
\hline LIPE & PNLIP & 0.033 & 0.783 & 0.533 & -0.267 & $\underline{0.317}$ & $\underline{-0.567}$ & $\underline{0.8}$ & 0.1 & $\underline{0.433}$ & $\underline{-0.5}$ & 0.0059 \\
\hline EP300 & GTF2H5 & $\underline{0.933}$ & -0.3 & 0.633 & -0.267 & 0.817 & -0.733 & 0.617 & -0.5 & 0.817 & -0.017 & $\underline{0.0024}$ \\
\hline EP300 & POLR2K & 0.817 & -0.35 & 0.783 & -0.117 & 0.8 & -0.733 & $\underline{0.65}$ & -0.533 & 0.833 & -0.167 & $\underline{0.0094}$ \\
\hline UBE2D2 & UBXN7 & 0.467 & -0.367 & 0.467 & -0.267 & 0.633 & -0.533 & 0.617 & -0.033 & $\underline{0.717}$ & -0.15 & 0.0068 \\
\hline ALMS1 & CEP290 & $\underline{-0.683}$ & $\underline{0.25}$ & $\underline{-0.617}$ & $\underline{0.567}$ & $\underline{-0.717}$ & $\underline{-0.05}$ & $\underline{0.733}$ & $\underline{-0.617}$ & $\underline{0.783}$ & $\underline{\underline{-0.05}}$ & $\underline{0.0047}$ \\
\hline RSRC1 & $\underline{\text { SNU13 }}$ & $\underline{-0.683}$ & $\underline{0.433}$ & $\underline{-0.683}$ & $\underline{0.433}$ & $\underline{\underline{-0.45}}$ & $\underline{0.333}$ & $\underline{-0.633}$ & $\underline{0.667}$ & $\underline{-0.217}$ & $\underline{0.717}$ & $\underline{0.0023}$ \\
\hline$\underline{\mathrm{BCR}}$ & CUX1 & $\underline{0.217}$ & $\underline{\underline{-0.5}}$ & 0.633 & -0.767 & $\underline{0.533}$ & $\underline{-0.533}$ & $\underline{0.35}$ & $\underline{-0.65}$ & 0.333 & -0.367 & $\underline{0.007}$ \\
\hline FGF21 & FLT1 & $\underline{0.6}$ & $\underline{-0.433}$ & $\underline{0.717}$ & $\underline{-0.517}$ & $\underline{0.183}$ & $\underline{-0.667}$ & $\underline{0.983}$ & $\underline{-0.417}$ & $\underline{0.833}$ & $\underline{-0.117}$ & $\underline{0.0014}$ \\
\hline DYNC1H1 & TUBGCP2 & -0.7 & $\underline{0.3}$ & -0.767 & $\underline{0.817}$ & $\underline{-0.767}$ & $\underline{0.317}$ & -0.55 & $\underline{0.3}$ & $\underline{-0.467}$ & $\underline{0.517}$ & $\underline{0.0006}$ \\
\hline CXCL9 & GRM4 & 0.1 & -0.85 & $\underline{0.05}$ & $\underline{-0.583}$ & $\underline{0.25}$ & $\underline{-0.5}$ & 0.767 & $\underline{-0.083}$ & $\underline{0.8}$ & $\underline{-0.167}$ & $\underline{0.0007}$ \\
\hline DYNC1H1 & PCNT & 0.483 & -0.75 & 0.4 & -0.933 & 0.267 & -0.65 & 0.567 & -0.6 & 0.4 & -0.367 & $\underline{0.004}$ \\
\hline CROCC & PCNT & -0.467 & 0.617 & -0.283 & 0.817 & -0.45 & 0.683 & -0.517 & 0.733 & -0.483 & 0.233 & 0.0079 \\
\hline IL2RA & $\underline{\mathrm{IL} 2 \mathrm{RB}}$ & $\underline{-0.5}$ & 0.317 & $\underline{-0.583}$ & $\underline{0.017}$ & $-\underline{-0.75}$ & $\underline{0.15}$ & $\underline{-0.783}$ & $\underline{0.517}$ & $\underline{-0.467}$ & 0.433 & $\underline{0.0072}$ \\
\hline MELK & RFC4 & -0.367 & 0.683 & $\underline{-0.117}$ & 0.533 & -0.633 & 0.617 & -0.033 & 0.583 & $\underline{-0.867}$ & 0.133 & $\underline{\underline{0}}$ \\
\hline $\begin{array}{l}\text { HNRNPA2B } \\
1\end{array}$ & $\underline{\mathrm{RBM} 17}$ & $\underline{-0.733}$ & $\underline{0.233}$ & $\underline{-0.767}$ & $\underline{0.4}$ & $\underline{-0.617}$ & $\underline{0.2}$ & $\underline{-0.683}$ & $\underline{0.017}$ & $\underline{-0.9}$ & $\underline{-0.183}$ & $\underline{0.0026}$ \\
\hline$\underline{\mathrm{ADCY} 3}$ & NUDT2 & $\underline{0.6}$ & $\underline{-0.267}$ & $\underline{0.483}$ & $\underline{-0.65}$ & $\underline{0.417}$ & $\underline{-0.667}$ & $\underline{0.65}$ & $\underline{-0.433}$ & $\underline{0.25}$ & $\underline{-0.517}$ & 0.0095 \\
\hline AGA & GCA & 0.817 & -0.317 & 0.817 & -0.133 & 0.8 & -0.65 & 0.617 & -0.617 & 0.633 & -0.45 & 0.0099 \\
\hline
\end{tabular}

Note: R: responders; NR: non-responders. The gene pairs fit two conditions: 1, The difference for Spearman correlation coefficient between responders and non-responders $>0.6$ and 2 , the corresponding p-value $<0.01$. 